\title{
WIRELESS SERVER FOR TOTAL HEALTHCARE SYSTEM FOR CLIENTS
}

\author{
Sajith.S.Nair ${ }^{1}$, Abdul NasirN.A ${ }^{2}$, Alan William ${ }^{3}$ \\ ${ }^{l}$ V.M.K.V. Engineering college Salem, Vinayaka Missions University, Salem, Tamilnadu \\ ${ }^{2}$ V.M.K.V. Engineering college Salem, Vinayaka Missions University, Salem, Tamilnadu \\ ${ }^{3}$ V.M.K.V. Engineering college Salem, Vinayaka Missions University, Salem, Tamilnadu
}

\begin{abstract}
This project is proposing a server for total monitoring of health conditions of clients / citizens including patients. The server start evaluating the health parameters of clients when he / she enters, the server area in each 40 seconds and stores the data at Base monitoring centre (BMC) and give alert when the medics software notice any variations in the clients health parameter and alerts both the client and the Base Monitoring Centre at the same time. By this project, we can terminate illness, Fatality etc. and by adopting this method, the clients can avoid waiting of time by visiting clinics for regular checkups, At the same time, it reduces the duty of doctors too.
\end{abstract}

Keywords: - Base Monitoring Centre (BMC), Sensors, Zigbee

\section{INTRODUCTION}

Here we are proposing this project not only for the patients; we are mainly targeting the clients / citizens of a nation from an area in this project. This proposing project will help our clients to avoid facing the critical stages like heart attack, stroke, high or low BP etc by the continues monitoring service did by our wireless server for total healthcare system. We are developing a residential based network for smart health care that will Open up new opportunities to the continues and long term monitoring of our clients. The sensors we are providing of wearable form and the some receivers and sensors placing at main and mini antennas in the environment. This project is mainly using Zig bee for data transfer from the sensors to the Base Monitoring Centre (BMC). The continuous updates of parameter helps the server to find out the small variations occurring in the body with the help of medic's software installed at the BMC and alerts our clients and the BMC at same time by using Zig bee. This helps our clients to get proper care to the health and move freely anywhere without any health worries. This project may create an era in the medical sector.

Our target is to improve the project an extend it to entire nation by adding more number of mini BMC and mini antennas, as the result, the mortality rate due to the negligence of health condition can be decreased and have a healthy citizens nation. The GSM based technology has been proposed here for the "wireless server for total healthcare system for clients". The system will alert the client and the base monitor centre before the client reaches the critical situation or before the health condition become abnormal.
Zig bee is the wireless sensor nodes mainly consists of the sensor unit, monitoring and controlling unit consists of microcontroller, XBee modules and with the help of Zig bee, the biosensors measures the medical parameters.

In this project, The Temperature, Heart rate, Oxygen saturation $\left(\mathrm{SpO}_{2}\right)$, Respiration rate are the parameters assigned to the system to monitor. These medical parameters are regularly collected from the clients and saved at BMC and it helps the doctor to check the medical parameters of our client at any time. Our clients are not at all restricted, they are free to move anywhere at any time, our server will start collecting the parameters when the client enters to the server area. Each client are identified by the unique ID provided to them, the receiver antenna at the BMC will first match the unique ID of the client and send the signals to the right clients profile. There the medic's software already programmed with normal parameters of that client matches with received parameters, the method we are proposing in our health care server.

\section{METHODOLOGY}

The Wireless sensor for total healthcare system for clients is working mainly depending on the Zig bee server. The Biosensor is used to measure Heart rate, Respiration rate, SPO2 and BP from the Client's body and co-ordinates all those signals and send to the BMC with the help of Zig bee server. The receiver module of Zig bee uses RS-232 serial port communication interference to send received signals to the PC at BMC. The collected parameters are verified with the medic's software installed in the BMC periodically in each 40 seconds and if any slight variation found, the medic's software 
will alert the both client and BMC at same time and helps to locate the location of the client too. Instantly the required steps need to be taken by the client will send automatically to the phone with the help of Zig bee server.
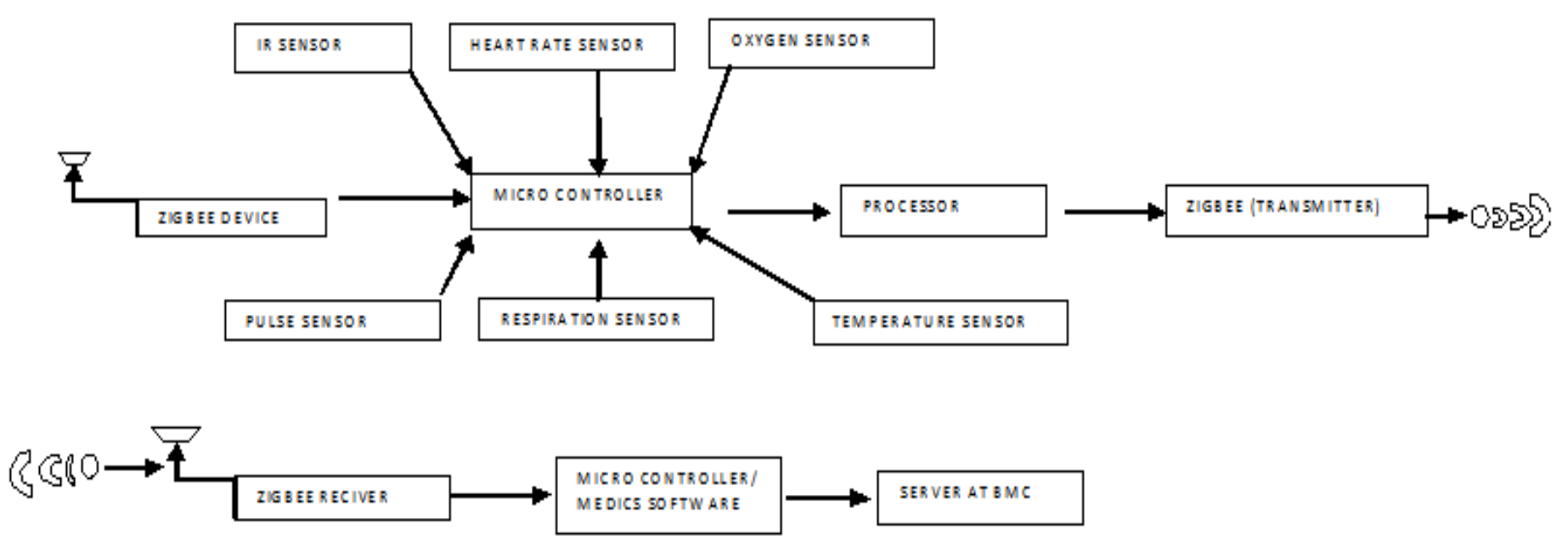

This block diagram gives an rough idea about the working principle of our server. The coordinator node collects the signal altogether and send to the micro controller. The micro controller receive the signals and use the $\mathrm{Zig}$ bee module to send all those signals to the Base Monitoring Centre ( BMC ). $\mathrm{Zig}$ bee is the network used here for the transmitting and receiving of signals.

Zig bee is a high level communication protocol with the normal frequency of $2.4 \mathrm{GHz}$. It is a two way communication system which can be able to transmit and receive signals within the targeted area. In one second 9600 bits of data can be sent and received. The range can be increased by connecting more Zig bee's altogether. One of the $802.15 .4 / \mathrm{Zig}$ bee modules can achieve a range up to $40 \mathrm{~km}$ and its range depends upon power and output. For additional safety, we use high range capacity "ad hoc wireless network " in towers and base centre for the transaction of data's in need of any emergency situation ,if any unavailability of network occurs due to any technical error. The high cost of installation and retrofit of monitoring system can be avoided by using ad hoc, self monitoring system.

The coordinator node collects all the details from all wireless sensors and sends to the base monitoring centre (BMC) using $\mathrm{Zig}$ bee module. The information send by $\mathrm{Zig}$ bee module is received by other Zig bee module at receiver module at BMC. Each coordinator node is identified by a unique ID used to identify each patient in the network. The body parameters from the each sensors have analog signals which has to be converted into digital signals by micro controller (PIC16F877A). The micro controller processes the signals and delivered to the central monitoring system by using a 433 $\mathrm{MHz}$ ASK RF transmitter through $433 \mathrm{MHz}$ ASK RF receivers which also consists of a microcontroller. The signals are again processed and the related information's are stored at data storage server at base centre.

The ARM micro controller will match the parameters already coded in medic's software at BMC, If any mismatch noticed, an alert occurs automatically to the client and the Base monitoring centre (BMC) at the same time.

The MICAZ MOTES embedded in the wearable form implemented to record activities such as Walking, Eating, Sitting, Moving etc and helps the Motion sensors to follow the clients.

The main 3 objectives for monitoring system in server:

- Study and analyse the micro controller and visual basic software in order to embed all these tools in system.

- Design wireless monitoring system using XBee module that can be easier to be monitor the patient.

- Design a friendly user to display the received data as the result of study.

The client monitoring unit with parameters such as Electrocardiogram, Movement Sensing, Temperature sensor etc consists of two units:

- Sensor functioning and collection of signals.

- Receiving the signals send to BMC.

This health monitoring system consisted of SEVERA sensors that we are going monitor. Then this data is send to the BMC through the SERVER.. The measure parameter data will be transferred through wireless node to Base Monitoring Centre (BMC ) and the evaluation of health condition of our clients are done. 


\section{HARDWARE REQUIREMENTS}

\section{Sensor and Transmission Unit}

- $\quad$ PIC16F877A

- Alarm System

- Temperature sensor

- Heart rate sensor

- $\mathrm{SpO}_{2}$ sensor

\section{Server PC}

- $\quad$ MPLAB IDE application for displaying the data

- $433 \mathrm{MHz}$ ASK RF Receiver

\section{SOFTWARE REQUIREMENTS}

- The various software requirements are:

- PROTEUS

- WIN PIC800

- MPLAB

- CCS Compiler

\section{OUTCOME AND ACHIEVEMENT}

- Helps the clients to find out small variations in their body.

- To avoid facing major critical stages like cardiac arrest, stroke etc.

- $\quad$ To keep clients with relaxed mind and better health

- $\quad$ Decrease Mortality rate due to diseases.

- Increase job opportunities.

\section{CONCLUSIONS}

The progress in science $\&$ technology is a non-stop process. New things and new technology are being invented. As the technology grows day by day, we can imagine about the future in which thing we may occupy every place.

The proposed system is more compact, user friendly and less complex, which can readily be used in order to perform. Though it is designed keeping in mind about the need in health sector, it can extended for other purposes such as commercial $\&$ research applications. Due to the probability of high technology used in this "Wireless Server for total healthcare system of clients" is fully software controlled with less hardware circuit. The feature makes this system is the base for future systems.

The principle of the development of science is that "nothing is impossible". So we shall look forward to a bright \& sophisticated world.

\section{FUTURE TRENDS}

- $\quad$ This project can be considered as a valuable milestone with the combined effect of Electronics and Communications fields. This project has wide applications in Health industries.

- To implement this project worldwide.

- To reduce the size of the sensor and try to make a single sensor which can sense all the parameters.

- This system can be modified more via internet and wide range networks. It is very simple and with affordable cost. This server can be designed very easily. This will give better relief to the Health Polyclinics that run on low budgets.

- It can be made more effective by using advance technologies and equipped devices. Centralized controlling is possible in this project.

\section{REFERENCES}

[1] Safaric S and Malaric K (2006) ZigBee wireless standard. In: Multimedia signal processing and communications, 48th Intl. Symp. ELMAR, Zadar, croatia (June).

[2] Ze Zhao and Li Cui (2005) EasiMed: A remote health caresolution. In: Proc. of the 2005 IEEE Engineering in medicine and Biology. 27th Ann. Conf., Shanghai China (September).

[3] 2009 International Conference on Advanced Information Networking and Applications Workshops "An RFID-based System for Emergency Health Care Services " by Cristina TURCU, Cornel TURCU, Valentin POPA

[4] Ms.Dharmistha D. Vishwakarma. IEEE 802.15.4 and Zigbee: A Conceptual Study, International Journal of Advanced Research in Computer and Communication Engineering, Vol. 1, Issue 7, September 2012

[5] XBee®/XBee-PRO® RF Modules, IEEE® 802.15.4 RF Modules by Digi International

[6] Zhao, G.; Mei, Z.; Liang, D.; Ivanov, K.; Guo, Y.; Wang, Y.; Wang, L. Exploration and implementation of a pre-impact fall recognition method based on an inertial body sensor network. Sensors 2012, 12, 15338 15355.

[7] Kumar, P.; Lee, S.-G.; Lee, H.-J. E-SAP: Efficientstrong authentication protocol for healthcare applications using wireless medical sensor networks. Sensors 2012, 12, 1625-1647

[8] Jin-Shyan Lee, Yu-Wei Su and Chung-Chou Shen. A Comparative Study of Wireless Protocols: Bluetooth, UWB, Zigbee, and Wi-Fi", The 33rd Annual Conference of the IEEE Industrial Electronics Society (IECON). 